\title{
ECG is not a reliable predictor of sudden cardiac death in the general population*
}

\author{
Juliane Theilade ${ }^{1,2 \#}$, Redi Pecini2 ${ }^{2 \# \dagger}$, Jacob L. Marott ${ }^{3}$, Gorm B. Jensen ${ }^{3,4}$ \\ ${ }^{1}$ The Danish National Research Foundation Centre for Cardiac Arrhythmia (DARC), Copenhagen, Denmark \\ ${ }^{2}$ Department of Cardiology, The Heart Centre, Copenhagen University Hospital-Rigshospitalet, Copenhagen, Denmark \\ ${ }^{3}$ Copenhagen City Heart Study, Bispebjerg Hospital, Bispebjerg, Denmark \\ ${ }^{4}$ Department of Cardiology, Copenhagen University Hospital, Hvidovre, Denmark \\ Email: ${ }^{\dagger}$ redpecini@me.com
}

Received 15 January 2013; revised 12 March 2013; accepted 14 April 2013

Copyright (C) 2013 Juliane Theilade et al. This is an open access article distributed under the Creative Commons Attribution License, which permits unrestricted use, distribution, and reproduction in any medium, provided the original work is properly cited.

\begin{abstract}
Objectives: To determine the predictive value of the ECG for sudden death in the general population. Design: In the Copenhagen City Heart Study, a randomly selected population sample in Copenhagen, Denmark has been followed prospectively since 1976. From this population sample, we analyzed ECGs of individuals who had suffered sudden cardiac death (SCD) before the age of 50 years and compared them with ECGs of a randomly selected control individuals from the same population sample. Specific ECG signs that could point toward a condition associated with a risk of SCD were noted. Results: From a total of 18,974 individuals in the cohort, 207 had died at an age younger than 50 years. Among these, 24 persons with SCD were identified. The most prevalent ECG abnormality was QRS fragmentation. We found no ECGs with long or short QTc, Brugada sign or WPW. The prevalence of signs of left ventricular hypertrophy, early repolarization, or fragmentation was not different from the prevalence of these signs in the control group. Conclusion: In the Copenhagen City Heart Study, the ECG failed to predict SCD in persons who died before the age of 50 years.
\end{abstract}

Keywords: Sudden Cardiac Death; ECG; Predictive Value of the ECG; General Population; Prediction of SCD; Arrhythmia; Cardiomyopathy; Ischemic Heart Disease; Copenhagen City Heart Study; Prospective

*This study was supported by a grant from the fondsbørsvekselerer Henry Hansen og hustrus legat. The Copenhagen City Heart Study is supported by the Danish Heart Association.

${ }^{\#}$ The first two authors have contributed equally in the manuscript.

${ }^{\dagger}$ Corresponding author.

\section{INTRODUCTION}

Coronary heart disease is by far the leading cause of the sudden cardiac death (SCD) and becomes more common as age advances [1]. In the young, hypertrophic cardiomyopathy (HCM), genetic arrhythmias and, to a lesser extent, Wolf-Parkinson-White syndrome (WPW), constitute a considerable proportion of the SCD causes [2-6]. Individuals with these diseases can be asymptomatic for a long time and SCD can be their first presentation of disease. For this reason, they are difficult to identify among the general population. Nevertheless, even in the asymptomatic persons, the ECG may show characteristic signs of HCM [7], long QT-syndrome [8], short QT-syndrome, arrhythmogenic right ventricular cardiomyopathy (ARVC) [9] Brugada syndrome [10], early repolarization, [11] or WPW [12]. As such, the ECG can identify individuals at risk for SCD. However, ECG screening in the general population is unproven and is only performed in specific groups and for specific purposes, such as employment, health insurance or participation in competitive sports. Therefore, the prevalence of characteristic ECG changes of diseases that are associated with SCD in young persons is not well studied. In the present study, we analyzed ECGs of individuals from the general population who had died at a young age, with particular emphasis on specific ECG changes that can point toward a disease associated with a risk of SCD.

\section{METHODS}

\subsection{Study Population}

The study population consisted of participants in the Copenhagen City Heart Study, details of which have been described earlier [13,14]. In brief, the Copenhagen City Heart Study is a prospective study of cardiovascular 
risk factors and diseases, which was started in 1976 with the invitation of a random population sample of healthy participants of either sex, aged 20 or above, from a well defined area of the city of Copenhagen. The first survey was carried out in 1976-1978. Of the 19,698 persons, who were invited to participate, 14,223 accepted the invitation. To date, three follow-ups of the Copenhagen City Heart Study have taken place. At each follow-up, the participants from the previous examinations, who were alive and accepted the invitation, underwent the examinations together with a supplement of new participants of young age. At the second examination in 1981-1983, 12,698 persons participated, at the third examination in 1991-1994, 10,135 participated, and at the fourth examination, 2001-2003, 6238 persons participated. At each examination, a 12-lead ECG was recorded in each participant. Follow-up was close to $100 \%$ by means of public registers.

Each person in Denmark is provided with a unique identification number upon birth or, if immigrant, upon the date of entry in Denmark. This identification number is used in the majority of public registers in order to identify and store data about a single person. Two of the public registers are the National Death Register, which, among other data, includes the cause of death and the National Patient Register. Each time a person in Denmark is hospitalized or is seen in an outpatient clinic, the diagnose(s) for the particular disease(s) that were the cause of the hospitalization or the visit are added to the National Patient Register. In the present study, we selected participants in the Copenhagen City Heart Study, who had died before the age of 50. Using the abovementioned public registers and the identification number we were able to track data about their cause of death and about their health status while alive. For each index case, we selected an age- and sex-matched control was selected from the same population sample. Controls were alive at least until the age of 50 .

\subsection{Definition of Sudden Cardiac Death}

We based the definition of sudden cardiac death on a combination of data from the National Patient Register and from the National Death Register. First, we selected all the individuals in the database of Copenhagen City Heart Study that had died before the age of 50 years. From these individuals we selected those, who had no records in the National Patient Register or had only records of non-serious diseases. Finally, we looked at the cause of death in the National Death Register for the remaining individuals. Those individuals with a diagnosis of sudden death not attributed to trauma, a cerebrovascular incident, suicide, drowning or drug overdose were selected for the ECG analyses. As such the defini- tion of SCD in our study was: individual died before the age of 50, with no records of serious disease and with no clear established non-cardiac death diagnosis. The cause of death in the death certificate was established by the patient's caring physician, the patient's primary physician, or by the physician, who performed the autopsy.

\subsection{ECG Analyses}

Two cardiology fellows (JT and RP) examined the ECGs together. In the individuals who had participated in more than one examination, the most recent ECG was chosen. We looked for specific ECG signs that could indicate increased risk for life threatening arrhythmias and sudden cardiac death. Regarding HCM, we used the Sokolow-Lyon criteria for left ventricular hypertrophy (sum of $\mathrm{S}$ wave in $\mathrm{V} 1$ and $\mathrm{R}$ wave in $\mathrm{V} 5$ or $\mathrm{V} 6 \geq 3.5 \mathrm{mV}$ ). We calculated the corrected QT interval (QTc) using the Bazett's formula (QT interval divided by the square root of the RR interval, both measured in seconds) [15]. As a sign of ARVC we only looked for an epsilon wave [9]. For signs of Brugada syndrome we looked for coved or saddleback configuration of the ST-segment in the leads V1, V2 and V3 [10]. For signs of WPW configuration we looked for a short PQ interval together with a delta wave. Finally, we looked for signs of fragmentation in the QRS complex defined as a slurring and slight prolongation of the final portion of the QRS complex without necessarily producing a broadening of the QRS complex [16]. Early repolarization was defined as an elevation of the $\mathrm{J}$ point at least $1 \mathrm{~mm}$ above the baseline level, either as QRS slurring (a smooth transition from the QRS segment to the ST segment) or notching (a positive J deflection inscribed on the S wave) in the inferior lead (II, III, and $\mathrm{aVF}$ ), lateral leads (I, aVL), and V3 to V6 [11]. Abnormal $\mathrm{T}$-waves were defined as negative, symmetric $\mathrm{T}$ waves, at least $3 \mathrm{~mm}$ in depth in all leads, except in leads III, aVR, V1 and V2.

In addition, we measured all the following intervals in milliseconds: RR, P wave width, PQ and QRS width. All the mentioned measurements from the case persons were compared with the measurements from the control persons. The two examiners had to agree whether a specific sign was present on the ECG. The intervals were measured separately, with each examiner measuring all of the ECGs.

The study was performed in a triple-blind fashion. During the ECG selection, ECG analyzes and the data processing the examiners were not aware of to which group the ECGs belonged.

\subsection{Statistical Analysis}

Continuous variables are presented as means \pm standard deviation (SD) and discrete variables as percent. For the 
comparison between continuous variables we used a two-sided $\mathrm{t}$ test. For the comparison between discrete variables we used the Fisher exact test. A two-sided $\mathrm{p}$ value of less than 0.05 was considered to indicate statistical significance.

\section{RESULTS}

Data about the selection mode of the deceased participants of the Copenhagen City Heart study, their death diagnose and past medical history are presented in Table 1 and Figure 1. As of June 1, 2010 we identified 207 persons that had died before the age of 50. 143 persons with records of chronic conditions in the National Patient Register were excluded. From the remaining 64 persons, 32 had no medical records about past diseases from hospitals or clinics in Denmark. The other 32 had medical records of non-chronic conditions. Examples of the latter were eye infection, appendicitis, fractures of the extremities, acute back pain. From this group of 64 persons, 24 individuals had suffered sudden death. The diagnoses in the death certificates or autopsy reports in these individuals were as follows: 8 ischemic heart disease, 13 SCD, 2 unspecified heart disease (ICD 8 code 429.9), and one unspecified acute heart failure (ICD 8 code 782.4).

Data about the latest ECG findings and measurements are presented in Table 2. No parameters were different between the case and the control groups. QRS fragmentation was the most frequent finding in the two groups. We found no WPW configuration and no Brugada sign in the ECGs. We found an epsilon wave configuration in only one of the ECGs, which belonged to a control person. The longest QTc interval was $471 \mathrm{~ms}$, the shortest was $360 \mathrm{~ms}$. Examples of the ECGs are presented in Figure 2.

The time of death from the first ECG registration varied from one to seven years.

\section{DISCUSSION}

We analyzed ECGs of persons, who had died suddenly before the age of 50 . These persons belonged to a large group chosen randomly from the general population, which has been followed prospectively since 1976. In the ECGs we looked specifically for signs that are related to conditions with increased risk for SCD. None of the ECGs signs were found more frequently in the case group compared to the control group. Different explanations may be possible for our findings.

First, we made the diagnosis of sudden death retrospectively, based on data from registries. Therefore, we

Table 1. Overview of general characteristics of individuals studied.

\begin{tabular}{lc}
\hline Persons died before the age of $\mathbf{5 0}$ & Number \\
\hline Total & 207 \\
Excluded persons & 143 \\
Known chronic conditions & \\
- Malignancy & 47 \\
- Alcohol or drug abuse & 38 \\
- Cardiovascular diseases $\quad$ Other & 36 \\
Trauma, suicide, homicide & 18 \\
No chronic conditions & 4 \\
Never hospitalized/seen in a clinic & 32 \\
\hline
\end{tabular}

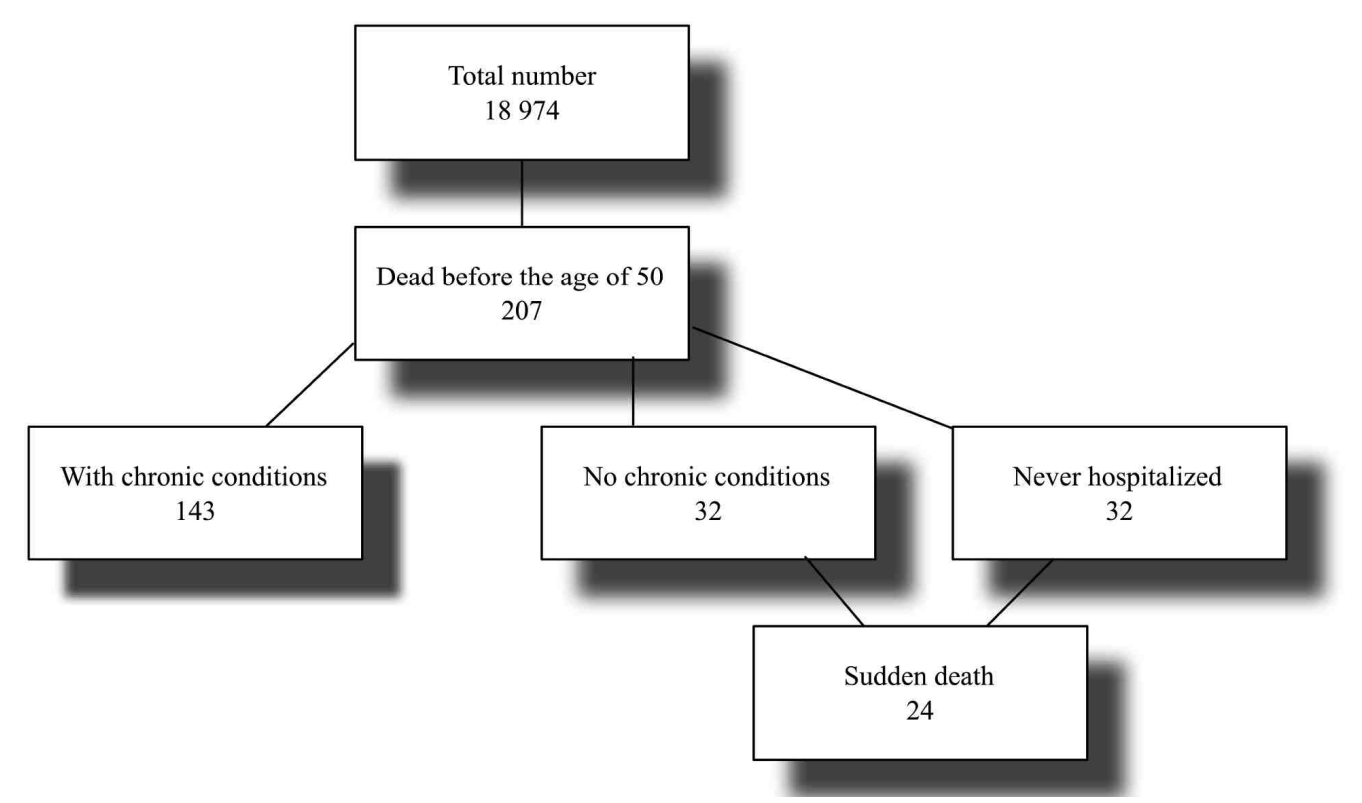

Figure 1. Algorithm of selection of individuals who died before the age of 50 years. 
Table 2. Comparisons between individuals with SCD and controls.

\begin{tabular}{lccc}
\hline \multicolumn{1}{c}{ Variable } & Case group & Control group & P value \\
\hline Age (years) & $45 \pm 5$ & $45 \pm 5$ & 0.9 \\
Male sex, n (\%) & $17(71)$ & $17(71)$ & 0.9 \\
Heart rate (bpm) & $77 \pm 18$ & $68 \pm 12$ & 0.05 \\
P wave width (ms) & $92 \pm 19$ & $95 \pm 24$ & 0.5 \\
PQ interval (ms) & $162 \pm 32$ & $171 \pm 19$ & 0.2 \\
QRS width (ms) & $93 \pm 11$ & $98 \pm 17$ & 0.3 \\
QTc interval (ms) & $416 \pm 27$ & $416 \pm 22$ & 0.9 \\
S-V1 + R-V5-6 (mV) & $29 \pm 8$ & $28 \pm 6$ & 0.3 \\
Fragmentation, n (\%) & $11(46)$ & $16(67)$ & 0.2 \\
Early repolarization, n (\%) & $2(8)$ & $6(25)$ & 0.2 \\
\hline
\end{tabular}

Early repolarization

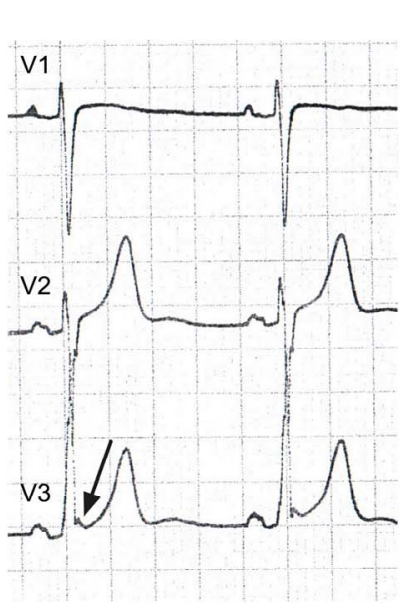

Epsilon wave

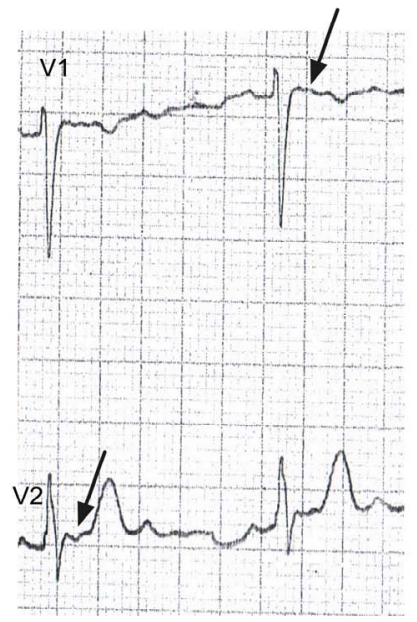

Fragmentation
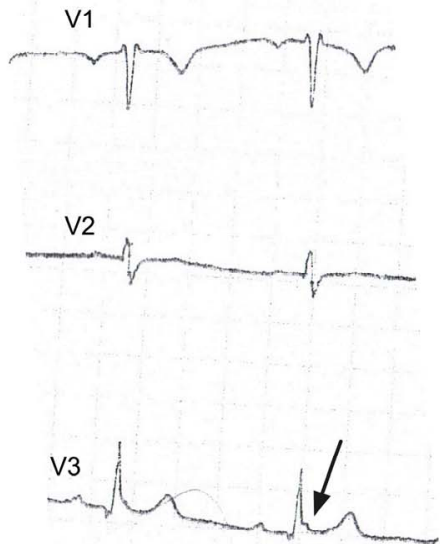

Figure 2. Examples of abnormal ECGs.

could not follow the definition of SCD strictly [1]. As such, it is possible that the individuals in our cohort might not have suffered cardiac death, but died from cerebrovascular causes. However, we believe that this problem was minimal, as none of the diagnoses in the death certificate described death from cerebrovascular cause. Furthermore, the prevalence of death from cardiac causes in individuals from 20 to 50 years old is about five times higher than the prevalence of death from cerebrovascular causes. [17]

Second, the age limit for inclusion in our cohort was 10 - 15 years higher than the age limit of most other SCD studies. This may have resulted in a greater number of SCD due to coronary heart disease, as this disease becomes more prevalent with increasing age. This is not surprising as even in studies, in which the age limit has been 35 - 40 years, coronary heart disease has been one of the most prevalent etiologies of SCD. [2-5,18] The higher age limit in our study was dictated by the low numbers of SCD in the younger participants. In our cohort, in the age groups younger than 40 we only found two persons with SCD.

Third, the low prevalence of ECG changes could be due to the fact that we only examined ECGs from persons that had died before the age of 50. It is possible that persons, who were alive in our cohort, may have shown ECG changes, which are characteristic for a genetic arrhythmia. However, this is of uncertain importance, as we tried to examine the value of ECG as a predictive tool for SCD in the general population. Besides being the cornerstone of diagnostic methods of genetic arrhythmias, the ECG also predicts the outcome of these diseases [19-21]. However, the predictive role of the ECG in these diseases comes from register studies. As such, 
these studies are biased towards patients with a worse prognosis. ECG failed to predict SCD in our cohort. Therefore, although ECG is invaluable in symptomatic patients, it may be unsuitable as a screening tool in large asymptomatic populations.

Most importantly, it is probable that the prevalence of the genetic arrhythmias was low in our cohort. Sudden death occurs most often in persons under the age of 40 in patients with genetic arrhythmias [19,21-23]. Nevertheless, we only found two persons with SCD before the age of 40 and, as such, the result may reflect the relative rarity of genetic arrhythmias in Denmark. This may particularly be true for the Brugada syndrome. In an analysis of the ECGs from the same cohort we found a lower prevalence of the Brugada sign than that reported from the majority of other studies [24]. Another recent study from Denmark also reported a lower number of HCM and ARVC than that reported in other studies in persons who had suffered SCD before the age of 35 [18].

\section{LIMITATIONS}

Autopsy was not performed systematically in all patients with sudden death. However, it is unlikely that a systematic autopsy would have influenced our results. A systematic autopsy might have resulted in the exclusion of some cases and the number of SCD in our cohort was already low from the start.

Not all the ECGs in our cohort were examined. Despite this limitation, the results of the study are still valid. The study was designed to look for ECG abnormalities in persons that had suffered SCD before the age of 50. Even if we had found abnormalities in the other ECGs, this would have been in persons who were alive after the age of 50. As such, the ECG would not have gained any further predictive value, as far as SCD is concerned.

\section{CONCLUSION}

In the Copenhagen City Heart Study, the ECG did not have a predictive value for SCD.

\section{REFERENCES}

[1] Priori, S.G., Aliot, E., Blomstrom-Lundqvist, C., Bossaert, L., Breithardt, G., Brugada, P., et al. (2002) Task force on sudden cardiac death, European society of cardiology. Europace, 4, 3-18. doi:10.1053/eupc.2001.0214

[2] Fragkouli, K. and Vougiouklakis, T. (2010) Sudden cardiac death: An 11-year postmortem analysis in the region of Epirus, Greece. Pathology-Research and Practice, 206, 690-694. doi:10.1016/j.prp.2010.05.005

[3] Doolan, A., Langlois, N. and Semsarian, C. (2004) Causes of sudden cardiac death in young Australians. Medical Journal of Australia, 180, 110-112.

[4] Puranik, R., Chow, C.K., Duflou, J.A., Kilborn, M.J. and
McGuire, M.A. (2005) Sudden death in the young. Heart Rhythm, 2, 1277-1282. doi:10.1016/j.hrthm.2005.09.008

[5] Drory, Y., Turetz, Y., Hiss, Y., Lev, B., Fisman, E.Z., Pines, A., et al. (1991) Sudden unexpected death in persons less than 40 years of age. American Journal of Cardiology, 68, 1388-1392.

doi:10.1016/0002-9149(91)90251-F

[6] Basso, C., Corrado, D., Rossi, L. and Thiene, G. (2001) Ventricular preexcitation in children and young adults: Atrial myocarditis as a possible trigger of sudden death. Circulation, 103, 269-275. doi:10.1161/01.CIR.103.2.269

[7] Savage, D.D., Seides, S.F., Clark, C.E., Henry, W.L., Maron, B.J., Robinson, F.C., et al. (1978) Electrocardiographic findings in patients with obstructive and nonobstructive hypertrophic cardiomyopathy. Circulation, 58, 402-408. doi:10.1161/01.CIR.58.3.402

[8] Monnig, G., Eckardt, L., Wedekind, H., Haverkamp, W., Gerss, J., Milberg, P., et al. (2006) Electrocardiographic risk stratification in families with congenital long QT syndrome. European Heart Journal, 27, 2074-2080. doi:10.1093/eurheartj/ehl159

[9] Marcus, F.I., McKenna, W.J., Sherrill, D., Basso, C., Bauce, B., Bluemke, D.A., et al. (2010) Diagnosis of arrhythmogenic right ventricular cardiomyopathy/dysplasia: proposed modification of the task force criteria. European Heart Journal, 31, 806-814.

doi:10.1093/eurheartj/ehq025

[10] Antzelevitch, C., Brugada, P., Borggrefe, M., Brugada, J., Brugada, R., Corrado, D., et al. (2005) Brugada syndrome: Report of the second consensus conference: Endorsed by the Heart Rhythm Society and the European Heart Rhythm Association. Circulation, 111, 659-670. doi:10.1161/01.CIR.0000152479.54298.51

[11] Haissaguerre, M., Derval, N., Sacher, F., Jesel, L., Deisenhofer, I., de Roy, L., et al. (2008) Sudden cardiac arrest associated with early repolarization. The New England Journal of Medicine, 358, 2016-2023. doi:10.1056/NEJMoa071968

[12] Fitzsimmons, P.J., McWhirter, P.D., Peterson, D.W. and Kruyer, W.B. (2001) The natural history of Wolff-Parkinson-White syndrome in 228 military aviators: A longterm follow-up of 22 years. American Heart Journal, 142, 530-536. doi:10.1067/mhj.2001.117779

[13] Appleyard, M. (1989) The Copenhagen city heart study: Østerbroundersøgelsen: A book of tables with the data from the first examination (1976-1978) and a five-year follow-up (1981-1983). Scandinavian Journal of Social Medicine, 41, 1-160.

[14] Schnohr, P., Jensen, G., Lange, P., Scharling, H. and Appleyard, M. (2001) The Copenhagen city heart study. Tables with data from the third examination 1991-1994. European Heart Journal, 3, 1-83.

[15] Funck-Brentano, C. and Jaillon, P. (1993) Rate-corrected QT interval: Techniques and limitations. The American Journal of Cardiology, 72, 17B-22B. doi:10.1016/0002-9149(93)90035-B

[16] Das, M.K. and Zipes, D.P. (2009) Fragmented QRS: A predictor of mortality and sudden cardiac death. Heart Rhythm, 6, S8-S14. doi:10.1016/j.hrthm.2008.10.019 
[17] Heron, M. (2011) Deaths: leading causes for 2007. National Vital Statistics Reports, 59, 1-95.

[18] Winkel, B.G., Holst, A.G., Theilade, J., Kristensen, I.B., Thomsen, J.L., Ottesen, G.L., et al. (2010) Nationwide study of sudden cardiac death in persons aged 1-35 years. European Heart Journal, 32, 983-990.

[19] Priori, S.G., Schwartz, P.J., Napolitano, C., Bloise, R., Ronchetti, E., Grillo, M., et al. (2003) Risk stratification in the long-QT syndrome. The New England Journal of Medicine, 348, 1866-1874. doi:10.1056/NEJMoa022147

[20] Sauer, A.J., Moss, A.J., McNitt, S., Peterson, D.R., Zareba, W., Robinson, J.L., et al. (2007) Long QT syndrome in adults. Journal of the American College of Cardiology, 49, 329-337. doi:10.1016/j.jacc.2006.08.057

[21] Priori, S.G., Napolitano, C., Gasparini, M., Pappone, C., Della Bella, P., Giordano, U., et al. (2002) Natural history of Brugada syndrome: Insights for risk stratification and management. Circulation, 105, 1342-1347. doi: $10.1161 / \mathrm{hc} 1102.105288$
[22] Zareba, W., Moss, A.J., Schwartz, P.J., Vincent, G.M., Robinson, J.L., Priori, S.G., et al. (1998) Influence of genotype on the clinical course of the long-QT syndrome. Interna- tional long-QT syndrome registry research group. New England Journal of Medicine, 339, 960-965. doi:10.1056/NEJM199810013391404

[23] Tabib, A., Loire, R., Chalabreysse, L., Meyronnet, D., Miras, A., Malicier, D., et al. (2003) Circumstances of death and gross and microscopic observations in a series of 200 cases of sudden death associated with arrhythmogenic right ventricular cardiomyopathy and/or dysplasia. Circulation, 108, 3000-3005. doi:10.1161/01.CIR.0000108396.65446.21

[24] Pecini, R., Cedergreen, P., Theilade, S., Haunso, S., Theilade, J. and Jensen, G.B. (2010) The prevalence and relevance of the Brugada-type electrocardiogram in the Danish general population: data from the Copenhagen city heart study. Europace, 12, 982-986.

doi:10.1093/europace/euq077 